\title{
Absorbability of Highly Porous Aluminum Oxide Ceramics
}

\author{
Katalin Sinkó \\ Chemical Institute, Eötvös University, Budapest H-1117, Hungary
}

\begin{abstract}
Highly porous aluminum oxide ceramics were synthesized by various low energy consumption techniques. This work demonstrates the efficiency of the preparation technique to tailor the structure of the materials. The drying method was varied and applied in vacuum (freeze drying), under supercritical conditions, and at atmospheric pressure. The pore system and the absorbability can be significantly affected by the conditions of the drying process. The absorbability of porous materials was compared basically for organic compounds. The gas and liquid adsorption measurements were carried out on a gas chromatography and mass spectrometry instrument. The absorbability of several porous aluminum oxide materials was compared to the adsorption data of pure carbon.
\end{abstract}

Key words: High porosity, absorbability, aluminum oxide, drying process.

\section{Introduction}

Porous alumina materials are capable to intensively adsorb gas and liquid compounds and they are excellent thermal insulating materials over a large temperature range and possess various applications in the field of heterogeneous catalysis [1-4]. The aim of the present research work was to investigate the absorbability of highly porous aluminum oxide ceramics in the function of the preparation technique. The effect of porosity, surface and morphology was studied on the adsorption ability. The aluminum oxides were synthesized by various liquid-phase techniques: sol-gel and co-precipitation methods. New, fast, low energy consumption, and different from the typical literature descriptions [5-14] techniques were developed for the synthesis of porous aluminium oxide system. The drying processes were applied in vacuum (freeze drying), under supercritical conditions, and at atmospheric pressure. The pore system can be significantly affected by the conditions of the drying process. The application of supercritical conditions results in aerogels with nanopores. The

Corresponding author: Katalin Sinkó, Ph.D., assoc. professor, research field: materials chemistry. vacuum freezing leads to formation of cryogels with hierarchical pore structures. This structure is composed from macropores ordered into channel-like architecture and nanopores are embedded in the wall of macropores. The drying at atmospheric pressure yields xerogels with macroporous or mesoporous skeletons depending on the conditions.

We have compared the absorbability of these porous materials basically for gas and liquid organic compounds. The literature studies of the alumina oxide's adsorption concentrate rather on the assimilation of inorganic compounds [15-23]. The most frequent aim of the adsorption investigation is the removal of arsenic ions from drinking water [16-18]. Only several publications deal with the adsorption of organic compounds [24, 25] or drugs [26, 27].

The published processes for the improvement of the effectiveness of adsorption focus on the introduction of various elements or compounds into the adsorbents $[18,20,23]$. This work presents the enhancement of the absorbability by sufficient preparation techniques. The adsorption data were checked by values of the pure carbon. The gas and liquid adsorption 
measurements were carried out on a gas chromatography and mass spectrometry instrument.

\section{Materials and Methods}

\subsection{Preparation of Porous Aluminum Oxide Ceramics}

Preparation of aluminum oxide xerogels: We prepared a monolith alcogel system from inorganic $\mathrm{Al}$ salt $\left(\mathrm{Al} \quad\left(\mathrm{NO}_{3}\right)_{3} .9 \quad \mathrm{H}_{2} \mathrm{O}\right.$ at Aldrich) in an organic medium (1-propanol) at $75^{\circ} \mathrm{C}$ in first step [28]. The molar ratio of 1-propanol/Al salt was 20. No basic or chelating agent was applied in the process and the precipitation also could be avoided. Gel systems form directly from the initial solutions. The gelation took for $24 \mathrm{~h}$. In order to increase the porosity and the specific surface area, additional materials (acetic acid, citric acid, and urotropine) were applied in the gelation. The second step was the drying at ambient pressure at $100{ }^{\circ} \mathrm{C}$ for the evaporation of the solvent and at $500{ }^{\circ} \mathrm{C}$ to form the final structure and morphology. The other type of xerogel was produced by a routine co-precipitation method in the presence of ammonia $\left(\operatorname{xerogel}^{\mathrm{c}}\right)$. The drying conditions were the same in every case.

Preparation of aluminum oxide cryogels: The first main step of the newly developed method is the preparation of a monolith hydrogel system. The aluminum oxide hydroxide containing hydrogel is prepared from inorganic $\mathrm{Al}$ salt $\left(\mathrm{Al}\left(\mathrm{NO}_{3}\right)_{3} \cdot 9 \mathrm{H}_{2} \mathrm{O}\right.$ at Aldrich) in an organic medium (1-propanol) at $75^{\circ} \mathrm{C}$. The following composition was used in the experiments: 1 mole Al nitrate and 10 mole 1-propanol. The hydrolysis and the precondensation reactions are performed by refluxing at about $80{ }^{\circ} \mathrm{C}$ for $11 \mathrm{~h}$. To make the condensation complete, the organic solvent must be carefully evaporated. The viscous system is soluble in water, but at an $\mathrm{Al}$ concentration of about $10-15 \mathrm{wt} \%(0.7 \mathrm{M})$, the solution turns into an optically clear gel after several hours. The second step is the freeze drying of the monolith hydrogel (at $233 \mathrm{~K}$ in a Christ, Alpha 2-4 LD plus cryostat instrument) [29].
Preparation of aluminum oxide aerogels: Alumina alcogels can be prepared by one-step sol-gel method from $\mathrm{Al}$ alkoxide precursors. In order to control the hydrolysis rate of $\mathrm{Al}$ alkoxides and to hinder the precipitation, a complexing agent was used. In the present study, $\mathrm{Al}$ isopropoxide is provided as precursor and isopropanol as solvent. The dissolution and the hydrolysis of precursor were carried out at $70{ }^{\circ} \mathrm{C}$ in the presence of some water $\left(5 \mathrm{~mol} \mathrm{H}_{2} \mathrm{O} / \mathrm{Al}\right)$ in isopropanol and ethyl acetate, Etac (3-10 mol $\mathrm{Etac} / \mathrm{Al})$. The total gelation and aging time attained 12 $h$. In the supercritical drying, the alcohol content of the wet gels was exchanged with liquid $\mathrm{CO}_{2}$ for 3-4 days, the temperature increased up to $45{ }^{\circ} \mathrm{C}$ and the pressure to ca. 100 bar. The pressure of the supercritical extractor was slowly ( $5 \mathrm{bar} / \mathrm{h})$ reduced to ambient pressure. The heat treatment of alumina aerogels was performed at $500{ }^{\circ} \mathrm{C}$ for $2 \mathrm{~h}$ to remove the organic compounds and to obtain the final structure.

\subsection{Investigation Methods}

Morphology and pore size: The morphology and the pore size of the ceramic powders and pellets have been studied by an FEI Quanta 3D FEG SEM (scanning electron microscope). The SEM images were prepared by the Everhart-Thornley secondary ETD (electron detector), and its ultimate resolution is 1-2 $\mathrm{nm}$. Since the conductance of the particles investigated is high enough to remove the electric charge accumulated on the surface, the SEM images were performed in high vacuum without any coverage on the specimen surface. For the best SEM visibility, the particles were deposited on an HOPG (graphite) substrate surface.

Surface area and porosity: The surface area and porosity of ceramic samples have been characterized by $\mathrm{N}_{2}$ sorption analysis at $25{ }^{\circ} \mathrm{C}$ on an autosorb computer controlled surface analyzer (AUTOSORB-1, Quantachrome or ASAP 2010 Micrometrics). Each sample was degassed for 12 hours prior to analysis. 
Adsorption: Vapour phase is collected in gas sampling bag for analysis by GC-MS. Instruments: YL6500 GC-MS (Young Lin Instrument Co.) gas chromatography and mass spectrometry. All the gases flowed into the reactor through Teflon tubing. The gas mixtures diluted in helium were flowed through the reactor with a linear velocity ranging between 60 and $120 \mathrm{~cm} \cdot \mathrm{s}^{-1}$. GC parameters are: S/SL injector with split ratio of $1 / 10$; constant $\mathrm{He}$ flow of $2 \mathrm{~mL} \cdot \mathrm{min}^{-1}$; and the injector temperature of $250{ }^{\circ} \mathrm{C}$. The heat program of oven: $3 \mathrm{~min}$ at $50^{\circ} \mathrm{C}$; heating rate of $10^{\circ} \mathrm{C} \cdot \mathrm{min}^{-1}$ to $150{ }^{\circ} \mathrm{C}$; heating rate of $30^{\circ} \mathrm{C} \cdot \mathrm{min}^{-1}$ to $250{ }^{\circ} \mathrm{C} ; 30.17 \mathrm{~min}$ at $250^{\circ} \mathrm{C}$. The measuring time is $16.5 \mathrm{~min}$. The compounds were monitored by mass spectroscopy scan mode collecting from $50 \mathrm{~m} / \mathrm{z}$ to 500 $\mathrm{m} / \mathrm{z}$ using a temporal resolution of $60 \mathrm{~ms}$, an ionization energy of $70 \mathrm{eV}$, and an emission current of $1,000 \mu \mathrm{A}$.

\section{Experimental Results}

\subsection{Synthesis and Drying of Highly Porous Aluminum Oxide Systems}

The preparation technique, especially the drying method was varied in order to obtain porous materials with high specific surface area and good adsorption ability. Aluminum oxide hydroxide containing gel systems derived by our new sol-gel synthesis were dried in various conditions. The drying processes were applied at atmospheric pressure, in vacuum, and under supercritical conditions. The drying at atmospheric pressure results in xerogels with macropores or mesopores. The porosity strongly depends on the drying conditions. Xerogel ${ }^{\mathrm{a}}$ and $\mathrm{Xerogel}^{\mathrm{b}}$ were synthesized by sol-gel method in the presence of acetic acid (1) and citric acid with urotropine (2). Xerogel $^{c}$ was produced by a routine co-precipitation technique in the presence of $\mathrm{NH}_{3}$ basic agent. This technique is a low-cost procedure. The other drying technique was the freeze drying in vacuum. The gel system dried by freezing is called cryogel. This type of process yields hierarchical pore structure. The pore system consists of open anisotropic macropores (with diameter of $10 \mu \mathrm{m}$ ) and mesopores (with diameter of 8 $\mathrm{nm}$ ) embedded in the wall of macropores. The macropores are ordered into channel-like architecture. The size of macropores depends on the size of frozen solvent crystals. The latter size is determined essentially by the rate of freezing [29]. The application of supercritical conditions leads to formation of aerogels with nanopores. By this way, the highest specific surface area can be obtained owing to the nanoporous system. This technique is the most expensive. Fig. 1 represents the structure and morphology of xerogels, cryogels, and aerogels. Table 1 summarizes the porosity, the specific surface area data of alox samples and the activated carbon providing

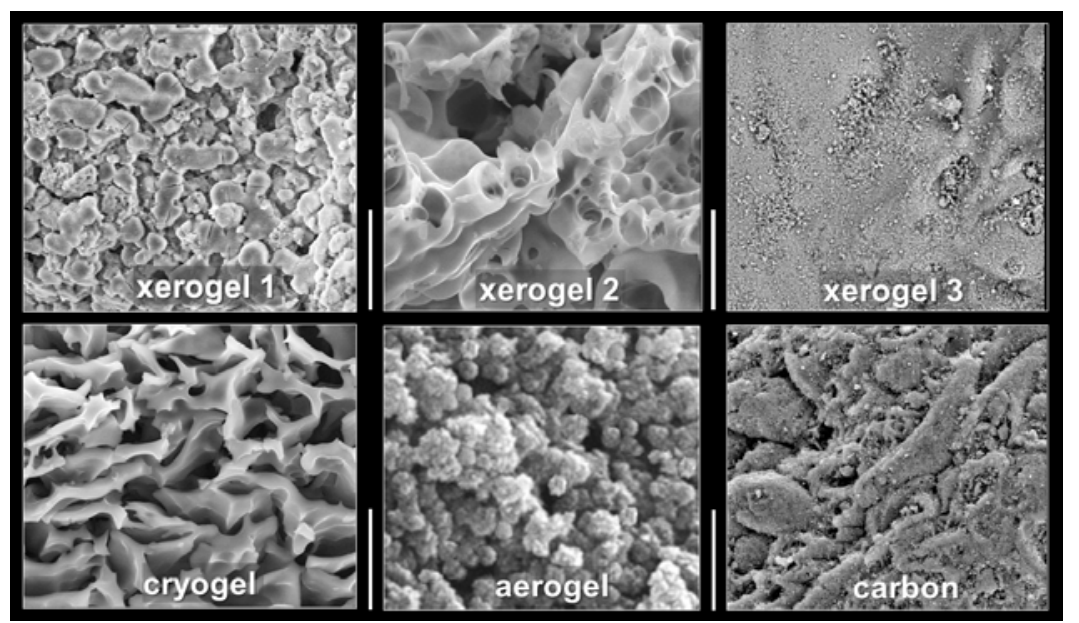

Fig. 1 SEM images of porous aluminum oxides. The magnifications are 1,000 $\mathrm{x}$, in the image of aerogel is $100,000 \mathrm{x}$. The line between the pictures indicates $50 \mu \mathrm{m}$ in every image exception in that of aerogel, where $50 \mathrm{~nm}$. 
the comparison.

The aerogel has the highest specific surface area $\left(500-520 \mathrm{~m}^{2} \cdot \mathrm{g}^{-1}\right)$ as expected due to its nanoporous system. The xerogel prepared by our new sol-gel technique and the cryogel can be characterized by more or less the same value of specific surface area $\left(\sim 300 \mathrm{~m}^{2} \cdot \mathrm{g}^{-1}\right)$ and by similar porous systems. Their porous systems are composed by two type of pore (10-15 $\mu \mathrm{m}$ and $<1 \mu \mathrm{m})$. The lowest value (130-160 $\left.\mathrm{m}^{2} \cdot \mathrm{g}^{-1}\right)$ has been measured in the sample of "xerogel" produced by a co-precipitation technique, this value is close to the literature data of xerogels (Table 1). The activated carbon has also high specific surface area $(>$ $\left.600 \mathrm{~m}^{2} \cdot \mathrm{g}^{-1}\right)$.

\subsection{Adsorption Investigations}

The adsorption ability depends not only on the pore system and specific surface area, but also on the chemical character. The porous aluminum oxide ceramics adsorb higher on average with $\sim 70 \%$ than activated carbon (Table 2). The good absorbability is owing to the strong Lewis acid character and the excellent complex formation of $\mathrm{Al}$ atoms/ions [30, 31]. The Al (III) efficiently bonds not only the inorganic but organic compounds/ligands, too. Many organic ligands can be connected to $\mathrm{Al}$ (III) as bidentate groups. The basic $\left(\mathrm{F}^{-}, \mathrm{CN}^{-}, \mathrm{AsO}_{4}{ }^{3-}\right.$, etc. $)$ and chelating agents (citric acid, acetic acid, EDTE, aliphatic or phenolic $\mathrm{OH}$-containing compounds, etc.) are very

Table 1 Porosity and specific surface data of aluminum oxide xerogel, cryogel, and aerogel systems.

\begin{tabular}{|c|c|c|c|c|c|c|c|}
\hline \multicolumn{2}{|l|}{ Samples } & \multicolumn{2}{|c|}{$\begin{array}{l}\text { Specific surface area } \\
\left(\mathrm{m}^{2} \cdot \mathrm{g}^{-1}\right)\end{array}$} & \multicolumn{2}{|c|}{$\begin{array}{l}\text { Porosity }^{* *} \\
(\%)\end{array}$} & \multicolumn{2}{|l|}{$\begin{array}{l}P_{\text {Pore size }}^{* *} \\
(\mathrm{~nm}-\mu \mathrm{m})\end{array}$} \\
\hline \multicolumn{2}{|l|}{ Xerogel $^{\mathrm{c}}$} & \multicolumn{2}{|c|}{$280-300$} & \multicolumn{2}{|l|}{$55-60$} & \multicolumn{2}{|l|}{$\begin{array}{l}10-15 \mu \mathrm{m} \\
0.5-1.0 \mu \mathrm{m}\end{array}$} \\
\hline \multicolumn{2}{|l|}{ Xerogel $^{\mathrm{d}}$} & \multicolumn{2}{|c|}{$240-260$} & \multicolumn{2}{|l|}{$60-70$} & \multicolumn{2}{|l|}{$5-30 \mu \mathrm{m}$} \\
\hline \multicolumn{2}{|l|}{ Xerogel $^{\mathrm{e}}$} & \multicolumn{2}{|c|}{$130-160$} & \multicolumn{2}{|l|}{$30-35$} & \multicolumn{2}{|l|}{$1-10 \mu \mathrm{m}$} \\
\hline \multicolumn{2}{|c|}{ Xerogel (mean literature data) } & \multicolumn{2}{|c|}{$100-150$} & \multicolumn{2}{|l|}{$20-40$} & \multicolumn{2}{|l|}{$1-15 \mu \mathrm{m}$} \\
\hline \multicolumn{2}{|c|}{ Cryogel } & \multicolumn{2}{|c|}{$280-310$} & \multicolumn{2}{|l|}{$70-80$} & \multicolumn{2}{|l|}{$\begin{array}{l}10-16 \mu \mathrm{m} \\
8-10 \mathrm{~nm}\end{array}$} \\
\hline \multicolumn{2}{|c|}{ Cryogel (mean literature data) } & \multicolumn{2}{|c|}{$200-300$} & \multicolumn{2}{|l|}{$40-70$} & \multicolumn{2}{|l|}{$10-100 \mu \mathrm{m}$} \\
\hline \multicolumn{2}{|l|}{ Aerogel } & \multicolumn{2}{|c|}{$500-520$} & \multicolumn{2}{|l|}{$60-65$} & \multicolumn{2}{|l|}{$10-20 \mathrm{~nm}$} \\
\hline \multicolumn{2}{|c|}{ Aerogel (mean literature data) } & \multicolumn{2}{|c|}{$300-700$} & \multicolumn{2}{|l|}{$50-80$} & $5-50 \mathrm{~nm}$ & \\
\hline Activated carbon & & 650 & & 40 & & - & \\
\hline $\begin{array}{l}\mathrm{N}_{2} \text { sorption measure } \\
\text { agent. }{ }^{\mathrm{d}} \text { The xerogel } \\
\text { co-precipitation techn }\end{array}$ & in th & resen & $\begin{array}{l}\text { SEM imag } \\
\mathrm{NH}_{3} \text { basic }\end{array}$ & The drying & ions were $\mathrm{t}$ & e in every c & $\begin{array}{l}\text { with acetic acid } \\
\text { ed by a routine } \\
\text { xerogels. }\end{array}$ \\
\hline Adsorpted & & & & Adsorptic & $\mathrm{g} / \mathrm{mg}$ adsorben & & \\
\hline compounds & Xerog & & Xerogel $^{\mathrm{b}}$ & Xerogel $^{\mathrm{c}}$ & Cryogel & Aerogel & Activated carbon \\
\hline Acetaldehyde & 0.395 & 0.2 & $0.256 \pm 0.2$ & $0.220 \pm 0.2$ & $0.595 \pm 0.2$ & $0.396 \pm 0.2$ & $0.092 \pm 0.1$ \\
\hline Acetone & 0.613 & \pm 0.2 & $0.315 \pm 0.2$ & $0.313 \pm 0.2$ & $1.050 \pm 0.3$ & $0.650 \pm 0.3$ & $0.122 \pm 0.1$ \\
\hline Acetic acid & 0.097 & $t 0.1$ & $0.038 \pm 0.1$ & $0.061 \pm 0.2$ & $0.358 \pm 0.1$ & $0.158 \pm 0.1$ & $0.047 \pm 0.1$ \\
\hline Benzene & 0.158 & \pm 0.1 & $0.102 \pm 0.1$ & $0.082 \pm 0.2$ & $0.213 \pm 0.1$ & $0.133 \pm 0.1$ & $0.032 \pm 0.1$ \\
\hline Methyl ethyl ketone & 0.011 & \pm 0.1 & $0.009 \pm 0.1$ & $0.007 \pm 0.1$ & $0.016 \pm 0.1$ & $0.013 \pm 0.1$ & $0.010 \pm 0.1$ \\
\hline Phenol & 0.666 & \pm 0.2 & $0.608 \pm 0.2$ & $0.503 \pm 0.2$ & $0.805 \pm 0.2$ & $0.701 \pm 0.2$ & $0.750 \pm 0.1$ \\
\hline Toluene & 0.169 & \pm 0.1 & $0.109 \pm 0.2$ & $0.082 \pm 0.1$ & $0.237 \pm 0.1$ & $0.202 \pm 0.1$ & $0.067 \pm 0.1$ \\
\hline$\sum$ condensates & 0.161 & \pm 0.02 & $0.152 \pm 0.02$ & $0.124 \pm 0.02$ & $0.185 \pm 0.02$ & $0.155 \pm 0.02$ & $0.092 \pm 0.01$ \\
\hline
\end{tabular}

${ }^{a}$ The xerogel was prepared by our new sol-gel technique with acetic acid agent. ${ }^{b}$ The xerogel was prepared by a sol-gel technique with citric acid and urotropine. ${ }^{c}$ Xerogel was produced by a routine co-precipitation technique in the presence of $\mathrm{NH}_{3}$ basic agent. The drying conditions were the same in every case of xerogels. 
effective for trivalent metal ions such as $\mathrm{Al}$ (III). As there are many publications, which are dealing with adsorption of inorganic compounds on $\mathrm{Al}$ (III) adsorbent, we investigated the connection of the organic components to aluminum oxide adsorbent. Apolar (e.g. benzene, toluene), polar (e.g. methyl ethyl ketone, acetic acid), aromatic (benzene, phenol, toluene), aliphatic (acetaldehyde, acetone), "oxo" (ketones, aldehydes), carboxylic (acids) organic compounds have been chosen for the adsorption investigations.

Among the porous aluminum oxide ceramics, the cryogel possesses the most effective absorbability (Table 2) regarding the adsorbed total condensates or the single gas compounds. The aerogel having the highest specific surface area can adsorb less than cryogel. The reason of that may be found in the too small pores, the molecules cannot so easily reach and connect to the nanopores than to macropores. The "xerogel" $\mathrm{l}$ " prepared by routine co-precipitation shows lower absorbability than the other xerogel samples synthesized by sol-gel chemistry. Between the additional materials, acetic acid is proved to be more efficient than citric acid or urotropine. However, the additional materials serve well their original purpose increasing the porosity and the specific surface area. But citric acid and urotropine blow up the structure and result in too large pores and smaller specific surface area.

If we only consider the absorbability data, the most effective porous ceramics is the cryogel. If we regard even the cost of the preparation technique beside the adsorption data, the best aluminum oxide ceramics for adsorption is "xerogel", which was synthesized by sol-gel route in the presence of acetic acid. The drying process can be performed at ambient pressure. The final heat treatment is carried out only at $500{ }^{\circ} \mathrm{C}$, this temperature provides the ceramics with a mixture of amorphous and crystalline $\left(\gamma-\mathrm{Al}_{2} \mathrm{O}_{3}\right)$ character. $\gamma-\mathrm{Al}_{2} \mathrm{O}_{3}$ has excellent adsorption ability $[15,32,33]$. This preparation is the lowest cost technique and results in the second best absorbability, thus the sol-gel derived "xerogel ${ }^{\mathrm{a}}$ " is the most promising and cost-effective adsorbent.

\section{Conclusions}

The aim of the present research work was to investigate the absorbability of highly porous aluminum oxide ceramics in the function of the preparation technique. The solvent-containing gel systems were synthesized by our new sol-gel method and a routine co-precipitation technique. The drying process was applied at atmospheric pressure, in vacuum, and under supercritical conditions. The drying at atmospheric pressure results in xerogels with macropores or mesopores. The freeze drying in vacuum yields cryogels with hierarchical pore structure. The application of supercritical conditions leads to formation of aerogels with nanopores.

We have compared the absorbability of these porous materials basically for gas and liquid organic compounds. The literature studies concentrate rather on the adsorption of inorganic compounds. In this work, the absorbability was enhanced by sufficient preparation techniques instead of typical published solution, i.e. any addition of various materials to the adsorbents. The adsorption data were compared to the values of the pure carbon. The cryogel samples present the best adsorption values owing to their suitable porous surface. However, the aerogels possess the highest specific surface area, but their nanoporosity is not so favorable for the organic adsorption. The porous aluminum oxide ceramics adsorb higher on average with $\sim 70 \%$ than commercially available activated carbon. Considering the cost of the preparation technique beside the adsorption data, the best aluminum oxide ceramic for adsorption is proved to be "xerogel". It was synthesized by sol-gel route in the presence of acetic acid and dried at atmospheric pressure and finally heat treated only at $500{ }^{\circ} \mathrm{C}$. This temperature provides the ceramics with a mixture of amorphous and crystalline $\left(\gamma-\mathrm{Al}_{2} \mathrm{O}_{3}\right)$ character. 


\section{Acknowledgments}

This study has been supported by OTKA K 115259 fund.

\section{References}

[1] Trimm, D. L., and Stanislaus, A. 1986. "The Control of Pore Size in Alumina Catalyst Supports." Appl. Catal. A 21: 215-38.

[2] Cejka, J. 2003. "Organized Mesoporous Alumina: Synthesis, Structure and Potential in Catalysis." Appl. Catal. A 254: 327-38.

[3] Richard, K. O. 1983. Applied Industrial Catalysis. Vol. 3. New York: Academic Press.

[4] Maki, T., and Sakka, S. 1988. "Preparation of Alumina Fibers by Sol-gel Method." J. Non-cryst. Solids 100: 303-8.

[5] Yoldas, B. E. 1975. "Alumina Gels that Form Porous Transparent $\mathrm{Al}_{2} \mathrm{O}_{3}$." J. Mater. Sci. 10: 1856-60.

[6] Yoldas, B. E. 1982. "Effect of Variations in Polymerized Oxides on Sintering and Crystalline Transformations." J. Am. Chem. Soc. 65: 387-93.

[7] Suh, D. J., Park, T. J., Kim, J. H., and Kim, K. L. 1997. "Fast Sol-gel Synthetic Route to High-surface-area Alumina Aerogels." Chem. Mater. 9: 1903-5.

[8] Pierre, A. C., Elaloui, E., and Pajonk, G. M. 1998. "Comparison of the Structure and Porous Texture of Alumina Gels Synthesized by Different Methods." Langmuir 14: 66-73.

[9] Rolison, D. R., and Dunn, B. J. 2001. "Electrically Conductive Oxide Aerogels: New Materials in Electrochemistry." J. Mater. Chem. 11: 963-80.

[10] Kureti, S., and Weisweiler, W. 2002. "A Novel Sol-gel Method for the Synthesis of $\Gamma$-Aluminium Oxide: Development of the Sol-gel Transformation and Characterization of the Xerogel." J. Non-cryst. Solids 303: 253-61.

[11] Carnes, C. L., Kapoor, P. N., Klabunde, K. J., and Bonevich, J. 2003. "Synthesis, Characterization, and Adsorption Studies on Nanocrystalline Aluminium Oxide and a Bimetallic Nanocrystalline Aluminium Oxide /Magnesium Oxide.” J. Chem. Mater. 13: 2922-9.

[12] Tewari, B. B., Shekar, S., Huang, L., and Wehmschulte, R. 2006. "Aluminumoxyhydride: Improved Synthesis and Application as a Selective Reducing Agent." Inorganic Chemistry 45: 2803-8.

[13] Passos, A. R., Pulcinelli, S. H., Briois, V., and Santelli, C. V. 2016. "High Surface Area Hierarchical Porous $\mathrm{Al}_{2} \mathrm{O}_{3}$ Prepared by the Integration of Sol-gel Transition and Phase Separation." RSC Advances 6: 57217-26.

[14] May, M., Navarrete, J., Asomoza, M., and Gomez, R.
2007. "Tailored Mesoporous Alumina Prepared from Different Aluminium Alkoxide Precursors." J. Porous Materials 14: 159-64.

[15] Bushey, T. J., and Dzombak, D. A. 2004. "Ferrocyanide Adsorption on Aluminum Oxides." J. Colloid and Interface Sci. 272: 46-51.

[16] Clifford, D. A. 1990. Ion Exchange and Inorganic Adsorption. AWWA, Water Quality and Treatment. 4th ed. New York: McGraw-Hill.

[17] Singh, P., Singh, T. S., and Pant, K. K. 2001. "Removal of Arsenic from Drinking Water Using Activated Alumina Research." Journal of Chemistry and Environment 5: 25-8.

[18] Liu, T., Wu, K., Xue, W., and Ma, C. 2015. "Characteristics and Mechanisms of Arsenate Adsorption onto Manganese Oxide-doped Aluminum Oxide." Environmental Progress and Sustainable Energy 34: 1009-18.

[19] Stietiya, M. H., and Wang, J. J. 2014. "Zinc and Cadmium Adsorption to Aluminum Oxide Nanoparticles Affected by Naturally Occurring Ligands." J. Environ Qual. 43: 498-506.

[20] Huang, C. P. 1977. "Removal of Phosphate by Powdered Aluminum Oxide Adsorption." Water Environment Federation 49: 1811-7.

[21] Ornelas-Lizcano, J. C., and Guirado-Lópeza, R. A. 2016. "Adsorption of Carbon Monoxide on Small Aluminum Oxide Clusters: Role of the Local Atomic Environment and Charge State on the Oxidation of the CO Molecule." J. Chem. Phys. 142: 124311-9.

[22] Bedemo, A., Chandravanshi, S. B., and Zewge, F. 2016. "Removal of Trivalent Chromium from Aqueous Solution Using Aluminum Oxide Hydroxide." Springer Plus 5: 1288-98.

[23] Salifu, A., Petrusevski, B., Ghebremichael, K., Modestus, L., Buamah, R., Aubry, C., and Amy, G. L. 2013. "Aluminum (Hydr) Oxide Coated Pumice for Fluoride Removal from Drinking Water: Synthesis, Equilibrium, Kinetics and Mechanism." Chem. Eng. J. 228: 63-74

[24] Laplante, F., Bouchard, N. A., Dubé, P., Ménard, H., and Brossard, L. 2003. "The Adsorption of Cyclohexanone on Aluminum Oxide-Hydroxide Powders in Relation to Its Electrocatalytic Hydrogenation." Canadian Journal of Chemistry 81: 1039-43

[25] Asmaly, H. A., Abussaud, B., Saleh, T. A., Laoui, T., Gupta, V. K., and Atieh, M. A. 2015. "Adsorption of Phenol on Aluminum Oxide Impregnated Fly Ash." Desalination and Water Treatment 57: 6801-8.

[26] Zhong, X., Zhang, H., Xiang, L., Royer, S., Valange, S., and Barrault, J. 2011. "Ultrasound-Assisted Adsorption Kinetics of Tetracycline on Aluminum Oxide." In 
Proceedings of the 3rd International CEMEPE \& SECOTOX Conference, Skiathos, 763-7.

[27] Chen, W. R., and Huang, C. H. 2010. "Adsorption and Transformation of Tetracycline Antibiotics with Aluminum Oxide." Chemosphere 79: 779-85.

[28] Sinkó, K., Kubuki, S., and Peterlik, H. 2013. "Various Three-Dimensional Structures Connected by Al-O/OH/Acetate-Al Bonds." Inorganic Chemistry 52: 13238-43.

[29] Sinkó, K., Kobzi, B., Sinclaire, J., Baris, A., and Temesi, O. 2015. "Influence of Cryogenic Drying Conditions on Hierarchical Porous Structure of Aluminum Oxide Systems." Microporous \& Mesoporous Materials 218: 7-14.
[30] Sposito, G. 1996. The Environmental Chemistry of Aluminum. 2nd ed. New York: Lewis Publishers.

[31] Lewis, T. E. 1989. Environmental Chemistry and Toxicology of Aluminum. New Orleans: CRC (Chemical Rubber Company) Press.

[32] Cheng, W. P., Huang, C., and Pan, J. R. 1999. "Adsorption Behavior of Iron-cyanide onto $\gamma-\mathrm{Al}_{2} \mathrm{O}_{3}$ Interface: A Coagulation Approach.” J. Colloid and Interface Sci. 213: 204-7.

[33] Andreev, N. S., Emeline, A. V., Polikhova, S. V., Ryabchuk, V. K., and Serpone, N. 2004. "Photoinduced Adsorption of Hydrogen and Methane on Gamma-alumina. The Photoinduced Chesorluminescence (PHICL) Effect.” Langmuir 6: 129-35. 